\title{
ON ULTIMATELY NONEXPANSIVE SEMIGROUPS
}

\author{
Michael Edelstein and Mo Tak Kiang
}

\begin{abstract}
A semigroup $G$ of continuous selfmappings of a metric space $(X, d)$ is called ultimately nonexpansive if for every $u, v$ in $X$ and $\alpha>0$, there is an $f$ in $G$ such that for all $g$ in $G, d(f g(u), f g(v)) \leqq(1+\alpha) d(u, v)$. It is shown that if $G$ is an ultimately nonexpansive commutative semigroup of selfmappings, then $G$ has a fixed point when any one of the following conditions is satisfied: (1) $X$ is a reflexive Banach space and each orbit under $G$ is precompact; (2) $X$ is a finite dimensional Banach space and there is a point in $X$ with a bounded orbit; (3) $X$ is a reflexive, locally uniformly convex Banach space having a point with a precompact orbit.
\end{abstract}

1. Introduction. Let $G$ be a family of selfmappings of a metric space $(X, d)$ and suppose that $G$ is a semigroup under composition. For any $x \in X, G(x)=\{g(x): g \in G\}$ is called the orbit of $x$ under $G$ and a point $z \in X$ such that $G(z)=\{z\}$ is called a fixed point of $G$. Fixed point properties of semigroups having various contractivity properties have been investigated by several writers (cf. [1], [3], [5], [6], [7], [8], [9], [10]). In [5], for example, an asymptotically nonexpansive semigroup $G$ (see definition given below), satisfying certain conditions, was shown to have a fixed point if $X$ is a finite dimensional Euclidean space and, for some $x \in X$, co $G(x)$ does not contain any affine flat of positive dimension. Similarly, in [8] a semigroup of selfmappings of a closed, bounded convex set in a uniformly convex Banach space-called eventually nonexpansive-was shown to have a fixed point provided certain additional hypotheses were satisfied.

In this paper we consider semigroups of mappings related to those of [8] but much more general; (e.g., members of the semigroup need not be lipschitzian).

Definition. A semigroup $G$ of continuous selfmappings of a metric space $(X, d)$ is called ultimately nonexpansive if for every pair of points $u, v \in X$ and for every $\alpha>0$, there is an $f^{*} \in G$ such that for all $g \in G$,

$$
d(f g(u), f g(v)) \leqq(1+\alpha) d(u, v)
$$

It is called asymptotically nonexpansive (cf. [5]) if (1) above is satisfied with $\alpha=0$.

It is the purpose of this paper to show that some of the fixed point theory for semigroups of nonexpansive mappings carries over to this much larger class of mappings. In particular this is the case 
when the orbits under $G$ and the space $X$ satisfy additional hypotheses; e.g., that some orbits be precompact and that $X$ be locally uniformly convex and reflexive. Thus if $X$ has the above property and at least one orbit is precompact then the existence of a fixed point follows.

2. Ultimately nonexpansive semigroups in general metric spaces. Certain properties of ultimately nonexpansive semigroups, some of them similar to those discussed in [5], and partially relying on the ideas and results of [5], are needed for the proof of our main results. We begin by reproducing the definition of " $G$-closure point" as given in [5].

Definition. The $G$-closure of $X$, where $(X, d)$ is a metric space, consists of all points $x$ in $X$ such that for some $z \in X$, any $\varepsilon>0$ and any $f \in G$ there is a $g \in G$ such that

$$
d(f g(z), x)<\varepsilon .
$$

The G-closure of $X$ is denoted by $X^{G}$ and it is a generalization of a similar notion for a single mapping as given in [2].

Proposition 1. Let $G$ be an ultimately nonexpansive commutative semigroup of selfmappings of a metric space $(X, d)$,

(a) if $x$ is a G-closure point, then $z$ can be replaced by $x$ in (2) (and any reference to $z$ can be deleted from the definition of $X^{G}$ );

(b) if $\mathrm{cl} G(x)$ is compact then it contains a G-closure point.

Proof. (a) Let $z \in X$ be such that for $\varepsilon>0$ and $f \in G$ there is a $g_{\varepsilon} \in G$ such that $d\left(f g_{\varepsilon}(z), x\right)<\varepsilon / 2(1+\varepsilon)$. Since $G$ is ultimately nonexpansive there is an $h_{\mathrm{\varepsilon}} \in G$ (corresponding to the points $g_{\mathrm{\varepsilon}} f(z)$, $x \in X$ and $\varepsilon$ ) such that for all $k \in G$,

$$
d\left(k h_{\varepsilon} g_{\varepsilon} f(z), k h_{\varepsilon}(x)\right) \leqq(1+\varepsilon) d\left(g_{\varepsilon} f(z), x\right)<\frac{\varepsilon}{2} .
$$

On the other hand, there is a mapping $t \in G$ (corresponding to $\left.h_{s} g_{s} f f \in G\right)$ such that

$$
d\left(f t h_{\varepsilon} g_{s} f(z), x\right)<\frac{\varepsilon}{2} .
$$

From (3) and (4) above we conclude that

$$
d\left(f t h_{\varepsilon}(x), x\right) \leqq d\left(f t h_{\varepsilon}(x), f t h_{\varepsilon} g_{\varepsilon} f(z)\right)+d\left(f t h_{s} g_{\varepsilon} f(z), x\right)<\varepsilon
$$

proving the assertion. 
(b) Suppose not. Then for every $u \in \operatorname{cl} G(x)$ there is an $\varepsilon_{u}>0$ and a $g_{u} \in G$ such that $d\left(g_{u} f(x), u\right) \geqq \varepsilon_{u}$ for all $f \in G$. The open balls $B\left(u, \varepsilon_{u}\right)$ form an open cover of cl $G(x)$. Hence there is a finite subcover of such balls with centers at $u_{1}, u_{2} \cdots, u_{n}$, say. Corresponding each $u_{i}, i=1, \cdots, n$, there is to a $g_{u_{i}} \in G$. Now $g_{u_{1}} \cdot g_{u_{2}} \cdots \cdot g_{u_{n}}(x)$ is in some $B\left(u_{i}, \varepsilon_{u_{i}}\right)$ contradicting the defining property of $g_{u_{i}}$; hence the result.

Proposition 2. Let $G$ and $(X, d)$ be as in Proposition 1. Then,

(a) if $x \in X^{G}$ then the restriction $\left.G\right|_{G(x)}$ of $G$ to $G(x)$ is a semigroup of isometries;

(b) if $\mathrm{cl} G(x)$ is compact then $\left.G\right|_{\mathrm{c} 1 G(x)}$ is a semigroup of isometries (of $\mathrm{cl} G(x)$ onto itself).

Proof. (a) Since each member of $G$ maps $G(x)$ into itself it is clear that $G_{\left.\right|_{G(x)}}$ is a semigroup again. Since the conclusion concerning isometries was obtained in [5] for asymptotically nonexpansive semigroups it suffices to show that $\left.G\right|_{G(x)}$ is such a semigroup. Suppose $\left.G\right|_{G(x)}$ fails to be asymptotically nonexpansive. Then, clearly, members $f_{1}(x), f_{2}(x)$ of $G(x)$ must exist such that for all $g \in G$ there is a $t \in G$ with

$$
\delta=d\left(t g f_{1}(x), t g f_{2}(x)\right)-d\left(f_{1}(x), f_{2}(x)\right)>0 .
$$

Let $\varepsilon=\delta\left(1+d\left(f_{1}(x), f_{2}(x)\right)\right)^{-1}$. From the definition of an ultimately nonexpansive semigroup it follows that corresponding to $\varepsilon$ and $f_{1}(x)$, $f_{2}(x)$ there is an $h \in G$ such that for all $f \in G$,

$$
d\left(f h f_{1}(x), f h f_{2}(x)\right) \leqq(1+\varepsilon) d\left(f_{1}(x), f_{2}(x)\right) .
$$

Hence,

$$
d\left(\operatorname{tgh} f_{1}(x), \operatorname{tgh} f_{2}(x)\right) \leqq(1+\varepsilon) d\left(f_{1}(x), f_{2}(x)\right) .
$$

Since $x \in X^{G}$ and each member in $G$ is continuous there is a $g_{0} \in G$ such that

$$
\max \left\{d\left(t g h g_{0} f_{i}(x), t g f_{i}(x)\right): i=1,2\right\}<\frac{\varepsilon}{2} .
$$

It follows that

$$
\begin{aligned}
d\left(t g f_{1}(x), t g f_{2}(x)\right) \leqq & d\left(t g f_{1}(x), t g h g_{0} f_{1}(x)\right)+d\left(t g h g_{0} f_{1}(x), t g h g_{0} f_{2}(x)\right) \\
& +d\left(t g h g_{0} f_{2}(x), t g f_{2}(x)\right) \\
< & \frac{\varepsilon}{2}+(1+\varepsilon) d\left(f_{1}(x), f_{2}(x)\right)+\frac{\varepsilon}{2} \\
= & d\left(f_{1}(x), f_{2}(x)\right)+\varepsilon\left(1+d\left(f_{1}(x), f_{2}(x)\right)\right) \\
= & d\left(t g f_{1}(x), t g f_{2}(x)\right)
\end{aligned}
$$


implying that $\delta=0$.

(b) This follows from (a) (and the fact that an isometry on a compact set is onto).

Proposition 3. Let $X$ and $G$ be as in Proposition 1. Then,

(a) if $u \in \operatorname{cl} G(x)$ with $x \in X^{G}$, and $v \in X, v \neq u$, there is a neighborhood $U$ of $u$ such that for any positive integer $n$ there exists an $f \in G$ with the property that for all $g \in G$ and $u^{\prime} \in U$,

$$
d\left(f g\left(u^{\prime}\right), f g(v)\right) \leqq\left(1+\frac{1}{n}\right) d\left(u^{\prime}, v\right) ;
$$

(b) if $\mathrm{cl} G(x)$ is compact, with $x \in X^{a}$, and $v \notin \mathrm{cl} G(x)$, then for any positive integer $n$, there is a $f \in G$ such that for all $g \in G$ and $u \in \operatorname{cl} G(x), d(f g(u), f g(v)) \leqq(1+1 / n) d(u, v)$.

Proof. (a) Let $f \in G$ be such that for all $g \in G, d(f g(u), f g(v)) \leqq$ $(1+1 / 4 n) d(u, v)$. Let $U=\left\{u^{\prime} \in \operatorname{cl} G(x): d\left(u^{\prime}, u\right)<(1 / 4 n) d\left(u^{\prime}, v\right)\right\}$. Then,

$$
\begin{aligned}
d\left(f g\left(u^{\prime}\right), f g(v)\right) & \leqq d\left(f g\left(u^{\prime}\right), f g(u)\right)+d(f g(u), f g(v)) \\
& \leqq d\left(u^{\prime}, u\right)+\left(1+\frac{1}{4 n}\right) d(u, v) \\
& <\frac{1}{4 n} d\left(u^{\prime}, v\right)+\left(1+\frac{1}{4 n}\right)\left(d\left(u^{\prime}, u\right)+d\left(u^{\prime}, v\right)\right) \\
& =\left(\frac{1}{4 n}+\left(1+\frac{1}{4 n}\right)^{2}\right) d\left(u^{\prime}, v\right) \leqq\left(1+\frac{1}{n}\right) d\left(u^{\prime}, v\right) .
\end{aligned}
$$

(b) Let $\left\{U_{i} \mid i=1,2, \cdots, k\right\}$ be a finite cover of cl $G(X)$ with the property that corresponding to each $U_{i}$ and for any positive integer $n$, there is an $f_{i} \in G$ such that for all $g \in G, u_{i} \in U_{i}$.

$$
d\left(f_{i} g\left(u_{i}\right), f_{i} g(v)\right) \leqq\left(1+\frac{1}{n}\right) d\left(u_{i}, v\right) .
$$

(Such a cover exists by (a) above and the compactness of $\mathrm{cl} G(x)$.) Let $f=f_{1} \circ f_{2} \circ \cdots \circ f_{k}$ then $f$ is as desired. Indeed, if $u \in \mathrm{cl} G(x)$ then $u \in U_{i}$ for some $i \in 1,2, \cdots, k$. Let $\hat{f}_{i}$ denote the product of $k-1$ members of $\left\{f_{1}, \cdots, f_{n}\right\}$ obtained by deleting $f_{i}$. Then

$$
\begin{aligned}
d(f g(u), f g(v)) & =d\left(f_{i} \hat{f}_{i} g\left(u_{i}\right), f_{i} \hat{f}_{i} g(v)\right) \leqq\left(1+\frac{1}{n}\right) d\left(u_{i}, v\right) \\
& =\left(1+\frac{1}{n}\right) d(u, v) .
\end{aligned}
$$

3. Centers of orbits. In this section $A$ will denote a nonempty closed convex subset of a Banach space $X$. Throughout, $G(x)$ will 
denote a precompact orbit under an ultimately nonexpansive semigroup of selfmappings of $X$.

In the proofs of the main results we shall make use of certain properties of Chebyshev centers of precompact orbits $G(x)$.

By a Chebyshev center $C_{A}$ of $G(x)$ with respect to $A$ we understand the subset of $A$ consisting of points $u$ such that the radius $R(u)$ of the smallest closed ball about $u$ containing $G(x)$ is minimal.

Thus, if

$$
R(u)=\sup \{\|u-g(x)\|: g \in G\}
$$

and

$$
R_{A}=\inf \{R(u): u \in A\}
$$

then

$$
C_{A}=\left\{u \in A: R(u)=R_{A}\right\} .
$$

The set $C_{A}$ is obviously also the intersection of closed balls about points of $G(x)$, each of radius $R_{A}$. Hence $C_{A}$ is closed convex and bounded; it is nonempty if $X$ is reflexive.

Lemma 1. If $G(x)$ is precompact, with $G(x) \neq\{x\}$, and $x \in X^{G}$, then $\operatorname{cl} G(x)$ and $C_{A}$ are disjoint for any closed convex subset $A$ of $X$.

Proof. Suppose not and let $u \in(\mathrm{cl} G(x)) \cap C_{A}$. Then $R(u)=R_{A}=$ $\operatorname{diam} G(x)$ and since members of $G$ act as isometries on $\operatorname{cl} G(x)$ it readily follows that $\operatorname{cl} G(x) \subset C_{A}$. This, in turn, implies that

$$
R(w)=R_{A}=\operatorname{diam} G(x)
$$

for all $w$ in $\overline{\text { co }} G(x)$, the closed convex hull of $G(x)$; against the known property of compact convex sets $K$ in a Banach space to contain a nondiametral point (i.e., a point $z$ such that $\sup \{\|z-w\|$ : $w \in K\}<\operatorname{diam} K)$.

Lemma 2. Let $C_{A}$ be the Chebyshev center of a precompact orbit $G(x)$, where $x \in X^{G}$, with respect to a closed convex set $A \subset X$. Suppose that $G(c)$ is containd in $A$ for some $c \in C_{A}$ and $\left\{f_{n}: n=1,2, \cdots\right\}$ has the property that

$$
\left\|f_{n} g(c)-f_{n} g(u)\right\| \leqq\left(1+\frac{1}{n}\right)\|c-u\|
$$

for all $u \in \operatorname{cl} G(x)$ and some $g \in G$. If $f_{n} g(c) \rightarrow \bar{c}$ then $G(\bar{c}) \subset C_{A}$.

Proof. Since $\left\{f_{n} g(c)\right\} \subset G(c) \subset A$ it suffices to show that for any $h, k \in G$ and any $\varepsilon>0$,

$$
\|k(\bar{c})-h(x)\|<R_{A}+\varepsilon .
$$


By continuity of $k$ there is a positive integer $n$ such that

$$
\left\|k(\bar{c})-f_{n} g k(c)\right\|<\frac{\varepsilon}{2} ;
$$

and since $h$ is an isometry on $\mathrm{cl} G(x)$ there is a $g_{n} \in G$ such that

$$
\left\|f_{n} g g_{n} \operatorname{kh}(x)-h(x)\right\|<\frac{\varepsilon}{2} .
$$

Hence

$$
\begin{aligned}
\|k(\bar{c})-h(x)\| \leqq & \left\|k(\bar{c})-f_{n} g k(c)\right\|+\left\|f_{n} g k(c)-f_{n} g g_{n} k h(x)\right\| \\
& +\left\|f_{n} g g_{n} k h(x)-h(x)\right\| \\
< & \frac{\varepsilon}{2}+\left(1+\frac{1}{n}\right)\left\|c-g_{n} h(x)\right\|+\frac{\varepsilon}{2} \\
\leqq & \left(1+\frac{1}{n}\right) R_{A}+\varepsilon .
\end{aligned}
$$

This being true for all $n=1,2, \cdots$, (5) follows.

Lemma 3. Let $C_{A}$ be the Chebyshev center of precompact orbit $G(x)$, where $x \in X^{a}$, with respect to a closed convex set $A \subset X$ and suppose that $c \in C_{A}$. If $G(c)$ is a precompact subset of $A$ then there is a $\bar{c} \in \mathrm{cl} G(c)$ such that $G(\bar{c}) \subset C_{A}$.

Proof. By Lemma 1, $c \notin \mathrm{cl} G(x)$. Hence, by Proposition 3(b), a sequence $\left\{f_{n} g(c)\right\}$ can be defined as in the preceding lemma. Since it is contained in $G(c)$, it contains a subsequence which converges to some $\bar{c} \in \operatorname{cl} G(x)$. Thus, after a suitable relabelling of the indices if required, the preceding lemma applies and the conclusion follows.

LemMa 4. Let $X$ be a strictly convex Banach space and suppose that, for some $x \in X, G(x)$ is precompact. Then the Chebyshev center $C$ of $G(x)$ with respect to $X$ consists of one point at most.

Proof. Suppose that $u, v \in C$ and let $w \in \operatorname{cl} G(x)$ be such that

$$
\left\|\frac{u+v}{2}-w\right\|=R_{X} .
$$

Then $2 R_{x}=\|u+v-2 w\| \leqq\|u-w\|+\|v-w\| \leqq 2 R_{x}$. Hence $\|u-w\|=\|v-w\|=R_{X}$. By strict convexity there is a $\lambda$ such that $u-w=\lambda(v-w)$. If $R_{X}=0$ there is nothing to prove (for then $G(x)=\{x\}=C)$. Otherwise, we have $|\lambda|=1$; and, again by $R_{X} \neq 0$, we must have $\lambda=1$. So $u=v$, proving the assertion. 
4. Main results.

THEOREM 1. Let $X$ be a reflexive Banach space and $G$ an ultimately nonexpansive commutative semigroup of selfmappings of $X$. If each orbit under $G$ is precompact then $G$ has a fixed point.

Proof. Let $C_{1}=X$ and $x_{1}$ be an arbitrary point in $X^{G}$. Suppose that for all ordinals $\mu<\lambda, C_{\mu}$ and $x_{\mu}$ are defined with $C_{\mu}$ closed convex and $G\left(x_{\mu}\right) \subset C_{\mu}$. If $\lambda$ is not a limit ordinal, i.e., if an immediate predecessor $\lambda-1$ exists, then $C_{\lambda-1}$ is defined and contains the precompact orbit $G\left(x_{\lambda-1}\right)$ with $x_{\lambda-1} \in X^{G}$. We define $C_{\lambda}$ as the Chebyshev center of $G\left(x_{\lambda-1}\right)$ and choose $x_{\lambda} \in C_{\lambda}$ such that $G\left(x_{\lambda}\right) \subset C_{\lambda}$. This is possible by Lemma 3, Proposition 3(b) and Proposition 2(b).

If $\lambda$ is a limit ordinal then

$$
C_{\lambda}=\bigcap\left\{C_{\mu}: \mu<\lambda\right\} .
$$

By reflexivity of $X, C_{\lambda} \neq \varnothing$. We proceed to show that an $x \in X^{G}$ exists such that $G(x) \subset C_{\lambda}$. Let $c \in C_{\lambda}$ be arbitrary. Then $c \in G_{\mu_{+1}}$ for any $\mu<\lambda$; so $c$ is in the Chebyshev center of $G\left(x_{\mu}\right)$ and Lemma 3 applies to the effect that a $\bar{c} \in \operatorname{cl} G(c)$ exists such that $G(\bar{c}) \subset G_{\mu_{+1}}$. Now cl $G(\bar{c})$ is also contained in $C_{\mu+1}$, and by compactness it contains a $\bar{c}_{\mu+1} \in X^{\sigma}$ such that $G\left(\bar{c}_{\mu_{+1}}\right) \subset \mathrm{cl} G(\bar{c}) \subset C_{\mu_{+1}}$. Let $c_{\lambda} \in \bigcap\left\{\operatorname{cl} G\left(\bar{c}_{\mu}\right): \mu<\lambda\right\} \subset$ $C_{\lambda}$. Then $\operatorname{cl} G\left(c_{\lambda}\right) \subset C_{\lambda}$ and a $\bar{c}_{\lambda} \in \operatorname{cl} G\left(c_{\lambda}\right)$ exists such that $\bar{c}_{\lambda} \in X^{\sigma}$. Choose $x_{\lambda}=\bar{c}_{\lambda}$. Thus a transfinite sequence of pairs $\left\{C_{\lambda}, x_{\lambda}\right\}$ arises. Since $C_{\lambda+1} \subset C_{\lambda} \backslash G\left(x_{2}\right)$ whenever $G\left(x_{2}\right)$ is not a singleton, a $\lambda^{*}$ (with $\operatorname{card} \lambda^{*} \leqq$ card $2^{X}$ ) must exist such that

$$
G\left(x_{\lambda^{*}}\right)=\left\{x_{\lambda^{*}}\right\},
$$

so $x_{\lambda^{*}}$ may serve as the desired fixed point.

THeORem 2. Let $X$ be a finite-dimensional Banach space and $G$ a commutative ultimately nonexpansive semigroup of selfmappings of $X$. If $G(y)$ is bounded for some $y \in X$ then $G$ has a fixed point.

Proof. Since cl $G(y)$ is compact it contains an $x \in X^{a}$. Let $C_{1}$ be the Chebyshev center of $G(x)$ with respect to $X$ and let $c_{1} \in C_{1}$. As in Lemma 2, with $A$ replaced by $X$, let $f_{n} \in G, n=1,2, \cdots$, be such that

$$
\left\|f_{n} g\left(c_{1}\right)-f_{n} g(u)\right\| \leqq\left(1+\frac{1}{n}\right)\left\|c_{1}-u\right\|
$$

for all $u \in \operatorname{cl} G(x)$ and some $g \in G$. (This is possible by Lemma 1 and Proposition 3(b).)

To apply Lemma 2 it suffices to show that a subsequence $\left\{f_{n_{i}} g\left(c_{1}\right)\right\}$ 
converges and to this end it is enough to check that $\left\{f_{n} g\left(c_{1}\right)\right\}$ is bounded. Now

$$
\begin{aligned}
& \left\|f_{n} g\left(c_{1}\right)-f_{m} g\left(c_{1}\right)\right\| \\
& \quad \leqq\left\|f_{n} g\left(c_{1}\right)-f_{n} g h(x)\right\|+\left\|f_{n} g h(x)-f_{m} g h(x)\right\|+\left\|f_{m} g h(x)-f_{m} g\left(c_{1}\right)\right\| \\
& \quad \leqq\left(1+\frac{1}{n}\right)\left\|c_{1}-h(x)\right\|+\operatorname{diam} G(x)+\left(1+\frac{1}{m}\right)\left\|c_{1}-h(x)\right\| \\
& \quad<\infty .
\end{aligned}
$$

Here $h$ is an arbitrary member of $G$. Suppose then that $\bar{c}_{1}$ is a cluster point of $\left\{f_{n} g\left(c_{1}\right)\right\}$. Then, by Lemma $2, G\left(\bar{c}_{1}\right) \subset C_{1}$.

Since $C_{1}$ is closed convex and bounded the center $C_{2}$ of $G\left(\bar{c}_{1}\right)$ with respect to $C_{1}$ exists and replacing $y$ and $X$ by $\bar{c}_{1}$ and $C_{1}$ respectively the preceding argument applies again to the effect that a $\bar{c}_{2}$ exists such that $G\left(\bar{c}_{2}\right) \subset C_{2}$. Proceeding inductively a sequence of centers $\left\{C_{n}\right\}$ and orbits $G\left(\bar{c}_{n}\right)$ lying within them arises, and it suffices to show that a positive integer $n \leqq \operatorname{dim} X$ exists such that $C_{n}=C_{n+1}$. To this end we note that $C_{1}$ clearly cannot contain an open subset and that, similarly $C_{k+1}$ cannot obtain any subset which is relatively open with respect to $C_{k}$ (unless $C_{k+1}=C_{k}$ ). Restated, either $C_{k+1}=C_{k}$ or $\operatorname{dim} \operatorname{sp} C_{k+1}<\operatorname{dim} \operatorname{sp} C_{k}$ where $\operatorname{sp} S$ denotes the affine span of the set $S \subset X$ and dim denotes the dimension.

Clearly then after $n \leqq \operatorname{dim} X$ steps we must arrive at a point $\xi$ such that $G(\xi)=\xi$.

THEOREM 3. Let $G$ be an ultimately nonexpansive commutative semigroup of selfmappings of a reflexive locally uniformly convex Banach space $X$ and suppose that $G(x)$ is precompact for some $x \in X^{G}$. Then the Chebyshev center of $G(x)$ is a singleton $c$ and $G(c)=\{c\}$; i.e., $c$ is fixed under $G$.

Proof. Since $X$ is strictly convex the Chebyshev center is a singleton $c$, by Lemma 4 . To show that $G(c)=\{c\}$ let $f_{n} \in G$ be such that, for all $u \in \operatorname{cl} G(x)$,

$$
\left\|f_{n} g(u)-f_{n} g(c)\right\| \leqq\left(1+\frac{1}{n}\right)\|u-c\|
$$

for a fixed $g \in G$ and all $n=1,2, \cdots$ (cf. Proposition $2(b)$ ). It suffices to show that $f_{n} g(c) \rightarrow c$.

Now

$$
\left\|f_{n} g(c)-u\right\| \leqq\left\|f_{n} g(c)-f_{n} g h(x)\right\|+\left\|f_{n} g h(x)-h(x)\right\|+\|h(x)-u\| .
$$

Choosing $g, h \in G$ so that $\|h(x)-u\|<(1 / 2 n) R_{X}$ and $\left\|f_{n} g h(x)-h(x)\right\|<$ $(1 / 2 n) R_{X}$, we obtain 


$$
\left\|f_{n} g(c)-u\right\| \leqq\left(1+\frac{2}{n}\right) R_{X}
$$

Hence

$$
\begin{aligned}
\left\|\frac{f_{n} g(c)+c}{2}-u\right\| & \leqq \frac{1}{2}\left\|f_{n} g(c)-u\right\|+\frac{1}{2}\|c-u\| \\
& \leqq \frac{1}{2}\left(1+\frac{2}{n}\right) R_{X}+\frac{1}{2}\|c-u\|
\end{aligned}
$$

Replacing $u$ by $u_{n} \in \operatorname{cl} G(x)$ such that

$$
\left\|z_{n}-u_{n}\right\|=R\left(z_{n}\right)
$$

where $z_{n}=\left(f_{n} g(c)+c\right) / 2$ then, whenever $z_{n} \neq c$,

$$
\begin{aligned}
R_{X}<R\left(z_{n}\right) & \leqq \frac{1}{2}\left(1+\frac{2}{n}\right) R_{X}+\frac{1}{2}\left\|c-u_{n}\right\| \\
& \leqq\left(1+\frac{1}{n}\right) R_{X} .
\end{aligned}
$$

It clearly follows that

$$
\left\|c-u_{n}\right\| \longrightarrow R_{X}
$$

Let $u_{n_{i}} \rightarrow \bar{u} \in \operatorname{cl} G(x)$ for some increasing sequence $\left\{n_{i}\right\}$. Then,

$$
\|c-\bar{u}\|=R_{X}
$$

and $\left\|f_{n} g(c)-\bar{u}\right\| \rightarrow R_{X}$. Set $w_{n}=\left(f_{n} g(c)-\bar{u}\right)\left(1-R_{X} /\left\|f_{n} g(c)-\bar{u}\right\|\right)$. Then $w_{n} \rightarrow 0$ and

$$
\left\|f_{n} g(c)-\bar{u}-w_{n}\right\|=R_{X}
$$

for all $n=1,2, \cdots$. Finally,

$$
\begin{aligned}
& \frac{1}{R_{X}}\left\|\frac{(c-\bar{u})+\left(f_{n} g(c)-\bar{u}-w_{n}\right)}{2}\right\| \\
& =\frac{1}{R_{X}}\left\|\left(\frac{c+f_{n} g(c)}{2}-\bar{u}\right)-\frac{w_{n}}{2}\right\| \rightarrow 1 .
\end{aligned}
$$

Since a Banach space is locally uniformly convex if, and only if, whenever members $y_{n}, x$ in it are such that $\left\|y_{n}\right\|=\|x\|=1$ and $\left\|y_{n}+x\right\| \rightarrow 2$ then $y_{n} \rightarrow x$, we conclude that

$$
f_{n} g(c)-\bar{u}-w_{n} \longrightarrow c-\bar{u}
$$

implying

$$
f_{n} g(c) \longrightarrow c
$$




\section{REFERENCES}

1. R. E. De Marr, Common fixed points for commuting contraction mappings, Pacific J. Math., 13 (1963), 1139-1141.

2. M. Edelstein, On nonexpansive mappings of Banach spaces, Proc. Camb. Phil. Soc., 60 (1964), 439-447.

3. - Fixed point theorems in uniformly convex Banach spaces, Proc. Amer. Math. Soc., 44 (1974), 369-374.

4. K. Goebel and W. A. Kirk, A fixed point theorem for asymptotically-nonexpansive mappings, Proc. Amer. Math. Soc., 35, No. 1 (1972), 171-174.

5. R. D. Holmes and P. P. Narayanaswami, On asymptotically-nonexpansive semigroups of mappings, Canad. Math. Bull., 13 (1970), 209-214.

6. R. D. Holmes and Anthony T. Lau, Asymptotically nonexpansive actions of topological semigroup and fixed points, J. London Math. Soc., (2) 5 (1972), 330-336.

7. M. T. Kiang, Fixed point theorems for certain classes of semigroups of mappings, Trans. Amer. Math. Soc., 189 (1974), 63-76.

8. — A fixed point theorem for eventually nonexpansive semigroups of mappings, J. Math. Analy. and Appl., 56 (1976), 567-569.

9. T. C. Lim, A fixed point theorem for families of nonexpansive mappings, Pacific J. Math., 53 (1974), 487-493.

10. T. Mitchell, Fixed points of reversible semigroups of nonexpansive mappings, Kodai Math. Sem. Rep., 22 (1970), 322-323.

Received December 12, 1980. The second author's research was supported by NSERC Grant no. A3999 and A8756.

Dalhousie University

HaLifax, N. S., Canada

AND

Saint Mary's University

Halifax, N. S., Canada 